\title{
ASYMPTOTIC STABILITY FOR SOME CRITICAL AUTONOMOUS DIFFERENTIAL EQUATIONS
}

\author{
ELLIOT WINSTON
}

\begin{abstract}
Liapunov functions are constructed and used to prove stability theorems for critical autonomous systems in which the linear part of the right-hand side has a zero eigenvalue.
\end{abstract}

1. Introduction. As an application of his celebrated "second method", Liapunov [3] proved the following theorem dealing with the critical case for an autonomous system of ordinary differential equations in which the linear part of the right-hand side has a zero eigenvalue.

THEOREM 1.1 (LiAPUNOv). Let $x$ be an n-vector, $y$ a scalar, and $A$ an $n \times n$ stable matrix. Consider the system of $n+1$ equations

$$
\begin{aligned}
& \dot{x}=A x+c\left(x_{i} x_{j}\right)+c\left(x_{i} y\right)+c\left(y^{m+1}\right), \\
& \dot{y}=b y^{m}+c\left(x_{i} x_{j} x_{k}\right)+c\left(x_{i} x_{j} y\right)+c\left(y^{m+1}\right),
\end{aligned}
$$

where $i, j, k=1,2, \cdots, n, m \geqq 2, b \neq 0$, and $c(z)$ represents collections of terms (vectors or scalars) which have a common factor of $z$. If $m$ is odd and $b<0$, then zero is uniformly asymptotically stable; otherwise, zero is unstable.

In this paper, we prove a stronger version of this theorem via Liapunov's method by using a function which is a modification of the one originally used by Liapunov; for the special case of two dimensions (when $n=1$ ), we obtain yet a more general result with a Liapunov function motivated by the following considerations. If $p_{y}(x, y)=q_{x}(x, y)$, then $p(x, y) d x+$ $q(x, y) d y$ is an exact differential, and hence the function

$$
W(x, y)=\int_{(0,0)}^{(x, y)} p(u, v) d u+q(u, v) d v
$$

is well defined because the line integral is independent of path. Moreover, curves defined by $W(x, y)=\alpha$, where $\alpha$ is a real parameter, are orthogonal to trajectories of

$$
\dot{x}=p(x, y), \quad \dot{y}=q(x, y) .
$$

Received by the editors June 26, 1973 and, in revised form, September 5, 1973.

AMS (MOS) subject classifications (1970). Primary 34D05, 34D20; Secondary 58F10, 93D05.

(c) American Mathematical Society 1974 
Thus, with suitable hypotheses on $p(x, y)$ and $q(x, y), W(x, y)$ becomes a candidate for a Liapunov function. For the more general situation in which the appropriate partial derivatives of $p(x, y)$ and $q(x, y)$ are not necessarily equal, we may consider the "completed" differential form

$$
\left[p(x, y)+\int_{0}^{y}\left(q_{x}(x, v)-p_{y}(x, v)\right) d v\right] d x+q(x, y) d y,
$$

which is always exact. If $q_{x}(x, y)-p_{y}(x, y)$ is small in some sense, we may hope that

$$
\begin{aligned}
V(x, y) & =\int_{(0,0)}^{(x, y)}\left[p(u, 0)+\int_{0}^{v} q_{x}(u, w) d w\right] d u+q(u, v) d v \\
& =\int_{0}^{x} p(u, 0) d u+\int_{0}^{y} q(x, v) d v
\end{aligned}
$$

defines a Liapunov function for (1.2). Other interesting constructions of Liapunov functions are discussed in LaSalle and Lefschetz [2] and Leighton [4].

2. Preliminaries. Let $R^{n}$ be Euclidean $n$-space with norm $|\cdot|$; if $x \in R^{n}$, denote the transpose of $x$ by $x^{T}$. Let $F: D \rightarrow R^{n}$, where $D$ is some domain in $R^{n}$ containing the origin, and assume that solutions of the autonomous differential equation

$$
\dot{x}=F(x)
$$

exist and are unique. Let $F(0)=0$ so that the zero function is a solution of (2.1). If $\phi(t, \tau, \xi)$ represents the solution of (2.1) such that $\phi(\tau, \tau, \xi)=\xi$, assume that $\phi(t, \tau, \xi)$ exists for $t \geqq \tau$ for all $\tau \geqq 0$ and sufficiently small $|\xi|$.

The various stability definitions of the zero solution of (2.1) can be found in LaSalle and Lefschetz [2], along with the basic ideas of Liapunov theory. In particular, the proofs of $\$ 3$ rest on Theorems II and III of that book.

3. Results. The following simple lemma is well known and can be found in Hale [1].

Lemma 3.1. Suppose $A$ is a real $n \times n$ matrix. The matrix equation $A^{T} B+B A=-C$ has a positive definite solution matrix $B$ for every positive definite matrix $C$ if and only if $A$ is a stable matrix, that is, the real parts of all the eigenvalues of $A$ are negative.

THEOREM 3.2. Let $A$ be be an $n \times n$ stable matrix, and consider the system of $n+1$ equations

$$
\begin{aligned}
& \dot{x}=A x+c\left(x_{i} x_{j}\right)+c\left(x_{i} y\right)+c\left(y^{m+1}\right), \\
& \dot{y}=b y^{m}+c\left(x_{i} x_{j}\right)+c\left(x_{i} y\right)+c\left(y^{m+1}\right),
\end{aligned}
$$


where $i, j=1,2, \cdots, n, m \geqq 2$, and $b \neq 0$. If $m$ is odd and $b<0$, then the zero solution of (3.1) is uniformly asymptotically stable; otherwise, zero is unstable.

Proof. Let $C$ be a positive definite matrix with all positive components such that $x^{T} C x \geqq 2|x|^{2}$; let $B$ be a positive definite matrix such that $A^{T} B+B A=-C$. Define

$$
\begin{aligned}
\quad V(x, y)= & x^{T} B x-b y^{m+1} /(m+1) \\
\Rightarrow \quad \dot{V}(x, y)= & \dot{x}^{T} B x+x^{T} B \dot{x}-b y^{m} \dot{y} \\
= & \left(x^{T} A^{T}+c^{T}\left(x_{i} x_{j}\right)+c^{T}\left(x_{i} y\right)+c^{T}\left(y^{m+1}\right)\right) B x \\
& +x^{T} B\left(A x+c\left(x_{i} x_{j}\right)+c\left(x_{i} y\right)+c\left(y^{m+1}\right)\right) \\
& -b y^{m}\left(b y^{m}+c\left(x_{i} x_{j}\right)+c\left(x_{i} y\right)+c\left(y^{m+1}\right)\right) \\
\Rightarrow \quad-\dot{V}(x, y)= & x^{T} C x+b^{2} y^{2 m}+c\left(x_{i} x_{j} x_{k}\right) \\
& +c\left(x_{i} x_{j} y\right)+c\left(x_{i} y^{m+1}\right)+c\left(y^{2 m+1}\right) .
\end{aligned}
$$

Since $C$ has positive components, the quadratic form $x^{T} C x$ dominates the terms $c\left(x_{i} x_{j} x_{k}\right)$ and $c\left(x_{i} x_{j} y\right)$ for small $|x|$ and $|y|$ so that

$$
-\dot{V}(x, y) \geqq x^{T} \hat{C} x+b^{2} y^{2 m}+c\left(x_{i} y^{m+1}\right)+c\left(y^{2 m+1}\right),
$$

where $\hat{C}$ is a positive definite matrix with positive components such that $x^{T} \hat{C} x \geqq|x|^{2}$. Hence, for small $|x|$ and $|y|$,

$$
\begin{aligned}
-\dot{V}(x, y) & \geqq|x|^{2}+\theta b^{2} y^{2 m}-2 M|x||y|^{m+1} \\
& =\left(|x|-M|y|^{m+1}\right)^{2}+\left(\theta b^{2}-M^{2} y^{2}\right) y^{2 m},
\end{aligned}
$$

where $M>0$ and $\theta=1^{-}$. Thus, $-\dot{V}(x, y)$ is positive definite in a neighborhood of the origin. Finally, if $b<0$ and $m$ is odd, $V(x, y)$ is positive definite; otherwise, $-b y^{m+1}$ is negative for certain arbitrarily small values of $y$, and therefore, $V(x, y)$ is negative arbitrarily near the origin along the $y$-axis.

The next theorem is concerned with the two-dimensional case.

\section{THEOREM 3.3. Consider}

$$
\begin{aligned}
& \dot{x}=a x+d y^{m}+c\left(x^{2}\right)+c(x y)+c\left(y^{m+1}\right), \\
& \dot{y}=b y^{m}+c\left(x^{2}\right)+c(x y)+c\left(y^{m+1}\right),
\end{aligned}
$$

where $a \neq 0, b \neq 0$, and $m \geqq 2$. If $m$ is odd, $a<0$ and $b<0$, then the zero solution of (3.2) is uniformly asymptotically stable; otherwise, zero is unstable. 
Proof. Define

$$
\begin{aligned}
-V(x, y)= & a x^{2} / 2+b y^{m+1} /(m+1)+d x y^{m} \\
\Rightarrow-\dot{V}(x, y)= & a x \dot{x}+b y^{m} \dot{y}+d\left(\dot{x} y^{m}+m x y^{m-1} \dot{y}\right) \\
= & a x\left(a x+d y^{m}+c\left(x^{2}\right)+c(x y)+c\left(y^{m+1}\right)\right) \\
& +b y^{m}\left(b y^{m}+c\left(x^{2}\right)+c(x y)+c\left(y^{m+1}\right)\right) \\
& +d\left(a x y^{m}+d y^{2 m}+c\left(x^{2} y^{m}\right)+c\left(x y^{m+1}\right)+c\left(y^{2 m+1}\right)\right. \\
& \left.+m b x y^{2 m-1}+c\left(x^{3} y^{m-1}\right)+c\left(x y^{2 m}\right)\right) \\
= & a^{2} x^{2}+b^{2} y^{2 m}+d^{2} y^{2 m}+2 a d x y^{m}+c\left(x^{3}\right) \\
& +c\left(x^{2} y\right)+c\left(x y^{m+1}\right)+c\left(y^{2 m+1}\right) .
\end{aligned}
$$

Hence, for small $|x|$ and $|y|$,

$$
\begin{aligned}
-\dot{V}(x, y) & \geqq \rho^{2} a^{2} x^{2}+\theta b^{2} y^{2 m}+d^{2} y^{2 m}-\left|2 \sigma a d x y^{m}\right| \\
& =\left(|\rho a x|-\left|\sigma d y^{m} / \rho\right|\right)^{2}+\left[\theta b^{2}+\left(1-\sigma^{2} / \rho^{2}\right) d^{2}\right] y^{2 m},
\end{aligned}
$$

where $\rho, \theta=1^{-}$and $\sigma=1^{+}$. Thus, the coefficient of $y^{2 m}$ is positive near the origin and $-\dot{V}(x, y)$ is positive definite. Now suppose $m$ is odd, $a<0$ and $b<0$. Then

$$
\begin{aligned}
V(x, y) & =|a| x^{2} / 2+|b| y^{m+1} /(m+1)-d x y^{m} \\
& =\left(\left(\frac{|a|}{2}\right)^{1 / 2} x-\frac{d y^{m}}{(2|a|)^{1 / 2}}\right)^{2}+\left(\frac{|b|}{m+1}-\frac{d^{2} y^{m-1}}{2|a|}\right) y^{m+1}
\end{aligned}
$$

so that $V(x, y)$ is positive definite; otherwise, $V(x, y)$ is negative arbitrarily near the origin along at least one of the coordinate axes.

Unfortunately, the author has not yet been able to answer the question of whether or not the preceding theorem has a natural extension in higher dimensions.

\section{REFERENCES}

1. J. K. Hale, Ordinary differential equations, Wiley, New York, 1969.

2. J. P. LaSalle and S. Lefschetz, Stability by Liapunov's direct method, with applications, Math. in Science and Engineering, vol. 4, Academic Press, New York, 1961. MR 24 \#A2712.

3. A. M. Liapunov, Problème général de la stabilité du mouvement, Ann. of Math. Studies, no. 17, Princeton Univ. Press, Princeton, N.J.; Oxford Univ. Press, London, 1947. MR 9, 34.

4. W. Leighton, On the construction of Liapunov functions for certain autonomous nonlinear differential equations, Contributions to Differential Equations 2 (1963), 367383. MR 27 \#3888.

Department of Mathematics, University of Maryland, Baltimore County, Catonsville, Maryland 21228 